\title{
State Extended Uncertainty Relations
}

\author{
D.A. Trifonov \\ Institute for Nuclear Research 72 Tzarigradsko chaussée, Sofia 1784, Bulgaria
}

November 16, 2018

\begin{abstract}
A scheme for construction of uncertainty relations (UR) for $n$ observables and $m$ states is presented. Several lowest order UR are displayed and briefly discussed. For two states $|\psi\rangle$ and $|\phi\rangle$ and canonical observables the (entangled) extension of Heisenberg UR reads $[\Delta p(\psi)]^{2}[\Delta q(\phi)]^{2}+[\Delta p(\phi)]^{2}[\Delta q(\psi)]^{2} \geq 1 / 2$. Some possible applications of the new inequalities are noted.
\end{abstract}

\section{Introduction}

The uncertainty principle is one of the basic principles in quantum physics. It was introduced by Heisenberg [1] on the example of the canonical observables $p$ and $q$, and rigorously proved by Kennard [1] in the form of the inequality $(\Delta p)^{2}(\Delta q)^{2} \geq 1 / 4$, where $(\Delta X)^{2}$ is the variance (dispersion) of $X$ (for the sake of brevity we work with dimensionless observables). This inequality is known as Heisenberg uncertainty relation (UR) for $p$ and $q$. It has been precised and extended to arbitrary two quantum observables by Schrödinger and Robertson [2] and to several observables by Robertson [3].

The Heisenberg UR became an irrevocable part of almost every textbook in quantum physics while the interest in the more precise Schrödinger [2] and Robertson [3] UR has grown up only recently in connection with the experimental generation of squeezed states of the electromagnetic field [4] and their generalization to arbitrary two and several observables [5, 6, 6, 8]. Robertson UR has been recently extended to all characteristic coefficients of the uncertainty matrix [9]. Extensions of the Heisenberg UR to higher moments of $p$ and $q$ are made in [10].

The UR listed above, and perhaps all the other ones so far considered, relate certain combinations of statistical moments of the observables in one quantum state. The main aim of the present paper is to extend the uncertainty principle to several states. The physical idea of such an extension is simple: one can measure and compare the statistical moments of two (or more) observables not only in one and the same state, but in two (or more) different states. The Hilbert space model of quantum mechanics permits us to derive easily such state extended UR. The extended UR can be divided into two classes - entangled $U R$ and nonentangled UR. An UR is called state entangled if it can not be factorized over distinct states.

Next we first recall the ordinary characteristic UR which include the known Schrödinger and Robertson ones (section 2) and then extend these UR to several states (section 2). Some other state extended UR are also established. Finally the simplest types of extended UR are displayed and discussed briefly (section 3).

\section{Characteristic UR}

The Schrödinger (or Schrödinger-Robertson)[2] UR for two observables $X$ and $Y$ reads

$$
(\Delta X)^{2}(\Delta Y)^{2}-(\Delta X Y)^{2} \geq \frac{1}{4}|\langle[X, Y]\rangle|^{2},
$$


where $\langle X\rangle$ is the mean value of $X$ in a given state, $(\Delta X)^{2}=\left\langle X^{2}\right\rangle-\langle X\rangle^{2}$ is the variance (the dispersion) of $X$, and $\Delta X Y \equiv\langle X Y+Y X\rangle / 2-\langle X\rangle\langle Y\rangle$ is the covariance of $X$ and $Y$. This UR was derived by Schrödinger from the Schwartz inequality for the matrix element $\langle\psi|(X-\langle X\rangle)(Y-$ $\langle Y\rangle)|\psi\rangle$. The less precise inequality $(\Delta X)^{2}(\Delta Y)^{2} \geq \frac{1}{4}|\langle[X, Y]\rangle|^{2}$ is usually called Heisenberg UR for $X$ and $Y$.

Robertson [3] has formulated the uncertainty principle for several observables $X_{1}, \ldots, X_{n}$ in terms of an inequality between determinants of the uncertainty matrix $\sigma(\vec{X} ; \psi)$ and the matrix $C(\vec{X} ; \psi)$ of the mean values of commutators of $X_{i}$ and $X_{j}$,

$$
\operatorname{det} \sigma(\vec{X} ; \psi)-\operatorname{det} C(\vec{X} ; \psi) \geq 0,
$$

where $\vec{X}=X_{1}, \ldots, X_{n}, \sigma_{i j}=(1 / 2)\left\langle X_{i} X_{j}+X_{j} X_{i}\right\rangle-\left\langle X_{i}\right\rangle\left\langle X_{j}\right\rangle$, and $C_{j k}=-(i / 2)\left\langle\left[X_{j}, X_{k}\right]\right\rangle$. For $n=2$ the inequality (2) recovers (1). Robertson has first proved the nonnegative definiteness of the matrix $R(\vec{X} ; \psi)$ (to be called Robertson matrix), $R(\vec{X} ; \psi)=\sigma(\vec{X} ; \psi)+i C(\vec{X} ; \psi) \geq 0$. This means that all principle minors of $R$ are nonnegative. For $n=2$ the inequality (2) coincides with $R(\vec{X} ; \psi) \geq 0$.

Robertson UR hold for mixed states $\rho$ as well (see e.g. the Appendix in [11]). Recently [9] the UR (2) has been extended to all characteristic coefficients $C_{r}^{(n)}(\sigma)$ of $\sigma(\vec{X} ; \rho)$ in the form

$$
C_{r}^{(n)}(\sigma) \geq C_{r}^{(n)}(C), \quad r=1,2, \ldots, n,
$$

where $C_{r}^{(n)}(C)$ is the characteristic coefficient of order $r$ of the mean commutator matrix $C(\vec{X} ; \rho)$. The characteristic coefficients of a matrix $M$ are defined by means of the secular equation [12] $\operatorname{det}(M-\lambda)=\sum_{r=0}^{n} C_{r}^{(n)}(M)(-\lambda)^{n-r}=0$. For $r=n$ one has $C_{n}^{(n)}(C)=\operatorname{det} C, C_{n}^{(n)}(\sigma)=\operatorname{det} \sigma$ and the characteristic UR (3) coincides with that of Robertson, eq. (2).

In comparison with the Heisenberg UR the Schrödinger and Robertson ones have the advantage of being covariant under linear nondegenerate transformations of the observables [8, 2]: if $\vec{X}^{\prime}=\Lambda \vec{X}$ then

$$
\sigma^{\prime}=\sigma\left(\vec{X}^{\prime} ; \rho\right)=\Lambda \sigma \Lambda^{T}, \quad C^{\prime}=C\left(\vec{X}^{\prime} ; \rho\right)=\Lambda C \Lambda^{T} .
$$

One sees that the equality in (2) is invariant under transformation (4) with nonsingular $\Lambda$, in particular with $\Lambda$ symplectic. For $r<n$ the equality in (3) is invariant under (位) if $\Lambda$ satisfies $\Lambda \Lambda^{T}=$ 1. If $X_{1}, \ldots, X_{n}$ close an algebra then Robertson UR is invariant under algebra automorphisms. In the case of orthogonal algebra the equality in (3) is invariant for every $r$.

The minimization of the characteristic UR and the relationship between the minimizing states and the group-related coherent states and squeezed states has been considered in 8, 9, 11], the minimizing states being called characteristic optimal uncertainty states or characteristic intelligent states of order $r$. States which minimize Schrödinger UR (1) were shown to be ideal squeezed states for $X$ and $Y$ [7, 8], while states which minimize (2) can be considered as squeezed states for several observables $[8$.

\section{State extended UR}

It should be useful first to recall that the derivation of the Robertson UR resorts to the following

Lemma 1 (Robertson). If $H$ is a nonnegative definite Hermitian matrix, then

$$
\operatorname{det} S-\operatorname{det} A \geq 0,
$$

where $S$ and $A$ are the real and the imaginary part of $H, H=S+i A$.

It is worth reminding that a matrix $H$ is nonnegative iff all its principle minors $M_{r}(H)$ are nonnegative,

$$
H \geq 0 \longleftrightarrow M_{r}(H) \geq 0, \quad r=1,2, \ldots, n .
$$


The proof of this lemma can be found in [3]. With minor changes in the notations it is reproduced in [11]. Robertson UR (22) corresponds to $H=R(\vec{X} ; \rho)$ in (5). In [9] this lemma was extended to all principle minors and to all characteristic coefficients of $H$,

$$
C_{r}^{(n)}(S)-C_{r}^{(n)}(A) \geq 0, \quad r=1,2, \ldots, n .
$$

The characteristic UR (3) correspond to $S=\sigma(\vec{X} ; \rho)$ and $A=C(\vec{X} ; \rho)$ in (畺).

The state extension of the ordinary UR, which we shall derive below, are based on the different physical choices of the matrix $H$ in (6), (5) and (7) and on the following lemma,

Lemma 2. If $H_{\mu}$ are nonnegative definite Hermitian $n \times n$ matrices, $\mu=1, \ldots, m$, then

$$
\begin{aligned}
C_{r}^{(n)}\left(S_{1}+\ldots+S_{m}\right)-C_{r}^{(n)}\left(A_{1}+\ldots+A_{m}\right) & \geq 0, \\
C_{r}^{(n)}\left(H_{1}+\ldots+H_{m}\right)-C_{r}^{(n)}\left(H_{1}\right)-\ldots-C_{r}^{(n)}\left(H_{m}\right) & \geq 0,
\end{aligned}
$$

where $S_{\mu}$ and $A_{\mu}$ are the real (and symmetric) and the imaginary (and antisymmetric) parts of $H_{\mu}$.

Proof. The validity of (8) immediately follows from the Robertson lemma and its extension (7), and the known fact that a sum of the Hermitian nonnegative matrices is a Hermitian nonnegative matrix. We proceed with the proof of the inequality (9). It is sufficient to establish it for $m=2$. Let $G$ and $H$ are Hermitian nonnegative definite matrices. We have to prove that $C_{r}^{(n)}(G+H)-C_{r}^{(n)}(G)-C_{r}^{(n)}(H) \geq 0$. Since the characteristic coefficients are sum of all principle minors [12] it is sufficient to consider the case of $r=n$, i.e. to prove the inequality $\operatorname{det}(G+H)-\operatorname{det} G-\operatorname{det} H \geq 0$.

(a) Let one of the two matrices (say $G$ ) is positive definite. Then both $G$ and $H$ can be diagonalized by means of a unitary matrix $U$ [12], $G^{\prime}=U^{\dagger} G U=\operatorname{diag}\left\{g_{1}, \ldots, g_{n}\right\}, H^{\prime}=U^{\dagger} H U=$ $\operatorname{diag}\left\{h_{1}, \ldots, h_{n}\right\}$, and

$$
\operatorname{det}(G+H)=\prod_{i}\left(g_{i}+h_{i}\right)=\prod_{i} g_{i}+\prod_{i} h_{i}+\Delta,
$$

where $\Delta=\operatorname{det}(G+H)-\operatorname{det} G-\operatorname{det} H=\operatorname{det}\left(G^{\prime}+H^{\prime}\right)-\operatorname{det} G^{\prime}-\operatorname{det} H^{\prime}, i=1, \ldots, n$,

$$
\Delta=g_{1} \prod_{j=2}^{n} h_{j}+g_{1} g_{2} \prod_{j=3}^{n} h_{j}+\ldots+h_{1} h_{2} \prod_{i=3}^{n} g_{i}+h_{1} \prod_{i=2}^{n} g_{i} .
$$

In view of $g_{i}>0$ and $h_{j} \geq 0$ all terms in (11) are nonnegative, thereby $\Delta \geq 0$.

(b) If both $G$ and $H$ are only nonnegative definite, then at least one $g_{i}$ and one $h_{j}$ are vanishing, that is $\operatorname{det} G=0=\operatorname{det} H$ and from nonnegativity of the sum $G+H \geq 0$ and (10) we obtain $\operatorname{det}(G+H)=\Delta \geq 0$. End of the proof.

Remark 1. From the above proof it follows that if $\operatorname{det} \sum H_{\mu}=\sum \operatorname{det} H_{\mu}$ then $\operatorname{det} H_{\mu}=0$, the inverse being not true.

Eqs. (8) and (9) can be called extended characteristic inequalities. They are invariant under the similarity transformation of the matrices $H_{\mu}$. At $m=1$ (one state) they recover the relations (7).

By a suitable physical choice of the nonnegative Hermitian matrices $H_{\mu}$ in the inequalities (6), (8), (9) one can obtain a variety of UR for several states and observables. We point out three physical choices of matrices $H_{\mu}$,

$$
\begin{gathered}
H=R(\vec{X} ; \rho)=\sigma(\vec{X} ; \rho)+i C(\vec{X} ; \rho) \quad \text { (Robertson matrix), } \\
\left.H=\Gamma\left(\chi_{1}, \ldots, \chi_{n}\right)=R(\vec{X} ; \vec{\psi}), \quad \| \chi_{k}\right\rangle=\left(X_{k}-\left\langle\psi_{k}\left|X_{k}\right| \psi_{k}\right\rangle\right)\left|\psi_{k}\right\rangle, \\
\left.H=\Gamma\left(\phi_{1}, \ldots, \phi_{n}\right)=G(\vec{X} ; \vec{\psi}), \quad \| \phi_{k}\right\rangle=X_{k}\left|\psi_{k}\right\rangle,
\end{gathered}
$$

where $\Gamma$ is the Gram matrix, $\Gamma_{i j}\left(\Phi_{1}, \ldots, \Phi_{n}\right)=\left\langle\Phi_{i}|| \Phi_{j}\right\rangle$, and $\left|\psi_{k}\right\rangle$ are normalized pure states. The diagonal elements $R_{i i}(\vec{X} ; \vec{\psi})$ and $R_{i i}(\vec{X} ; \rho)$ are just the variances of $X_{i}$ in the state $\left|\psi_{i}\right\rangle$ and 
(generally mixed) state $\rho$, while $G_{i i}=\Gamma_{i i}\left(\phi_{1}, \ldots, \phi_{n}\right)=\left(\Delta X_{i}\left(\psi_{i}\right)\right)^{2}+\left\langle\psi_{i}|X| \psi_{i}\right\rangle^{2}$. Therefore the inequalities obtained in the above scheme can be regarded as state extended $U R$. For brevity UR for $n$ observables and $m$ states should be called UR of type $(n, m)$.

For pure states $\left|\psi_{k}\right\rangle(12)$ is a particular case of (13), and the common structure of (12) - (14) is $H=\Gamma\left(\Phi_{1}, \ldots, \Phi_{n}\right)=T(\vec{X}, \vec{\psi})$, where $\Phi_{k}$ denote the corresponding nonnormalized states $\left|\Phi_{k}\right\rangle$. Let us note that $\Gamma\left(\Phi_{1}, \Phi_{2}\right) \geq 0$ is equivalent to the Schwartz inequality. For one observable $X$ the matrix $G(X, \vec{\psi})$ is covariant under linear transformation of states,

$$
\left|\psi_{i}^{\prime}\right\rangle=U_{i k}^{*}\left|\psi_{k}\right\rangle \quad \rightarrow \quad G\left(\vec{X}, \vec{\psi}^{\prime}\right)=U G(\vec{X}, \vec{\psi}) U^{\dagger} .
$$

This property entails the invariance of the equality in the extended highest order characteristic UR of type $(1, \mathrm{~m})$, constructed by means of $G(X, \vec{\psi})$. If $U U^{\dagger}=1$ then all order extended characteristic UR of type $(1, \mathrm{~m})$ are invariant. Compare this symmetry with that of ordinary characteristic UR under transformation (1). The extended UR of types (n,m) with $n, m>1$ do not possess such symmetry.

In all three cases of $H$ with pure states the UR $H \geq 0$ are disentangled by means of linear transformations of states. The UR corresponding to (9) and (8) are state entangled. The proof of the nonentangled character of the UR $H \geq 0$ for (12), (13) and (14) with pure states can be easily carried out using the diagonalization of $\Gamma=\Gamma\left(\Phi_{1}, \ldots, \Phi_{m}\right)$.

\section{Extended UR of simplest types}

UR of type $(\mathbf{1 , 2})$. For $m=2$ (two states) the choices $H=R(X ; \vec{\psi})$ and $H=G(X ; \vec{\psi})$ in (6), (8) and (9) produce two different UR which we write down as

$$
\begin{aligned}
& \left(\Delta X\left(\psi_{1}\right)\right)^{2}\left(\Delta X\left(\psi_{2}\right)\right)^{2} \geq\left|\left\langle\psi_{1}\left|\left(X-\left\langle\psi_{1}|X| \psi_{1}\right\rangle\right)\left(X-\left\langle\psi_{2}|X| \psi_{2}\right\rangle\right)\right| \psi_{2}\right\rangle\right|^{2}, \\
& \left(\left(\Delta X\left(\psi_{1}\right)\right)^{2}+\left\langle\psi_{1}|X| \psi_{1}\right\rangle^{2}\right)\left(\left(\Delta X\left(\psi_{2}\right)\right)^{2}+\left\langle\psi_{2}|X| \psi_{2}\right\rangle^{2}\right) \geq\left|\left\langle\psi_{1}\left|X^{2}\right| \psi_{2}\right\rangle\right|^{2} .
\end{aligned}
$$

Since the right hand sides of (15) and (16) are generally greater than zero these inequalities reveal correlations between the statistical second moments of $X$ in different states.

These two UR are independent in the sense that none of them is more precise than the other. To prove this suffice it to consider the example of $X=p$ and two Glauber coherent states. The minimization of (15) and (16) occurs iff the two nonnormalized states in the Gram matrix are proportional. In the case of (15) this is $(X-\langle 2|X| 2\rangle)\left|\psi_{2}\right\rangle=\lambda(X-\langle 1|X| 1\rangle)\left|\psi_{1}\right\rangle$ wherefrom we easily deduce that if $X$ is a continuous observable (such as $q, p$ or $p^{2}-q^{2}$ and $p q+q p$ ) then UR (15) is minimized iff $\left|\psi_{1}\right\rangle=\left|\psi_{2}\right\rangle$. It follows from this condition that (15) and (16) can be used for construction of distances between quantum states (observable induced distances) [11].

UR of type $(\mathbf{2 , 1})$. For $n=2$ the inequalities (9), (8) and (6) coincide. For two observables $X, Y$ and one state the Robertson choice (12) coincides with (13) and when replaced in (5) - (9) it produces Schrödinger UR (11). The choice (14) in (8) and (9) generates the invariant UR

$$
\left.\left.\left[(\Delta X)^{2}+\langle X\rangle^{2}\right)\right]\left[(\Delta Y)^{2}+\langle Y\rangle^{2}\right)\right] \geq(\Delta X Y+\langle X\rangle\langle Y\rangle)^{2}+\frac{1}{4}|\langle[X, Y]\rangle|^{2},
$$

which however can be shown to be less precise than the Schrödinger one (11). The interpretation of any UR of type $(2,1)$ is the same as that of Schrödinger UR.

UR of type $(\mathbf{2 , 2})$. The number of possible UR of type $(2,2)$ is much more. The inequalities $R\left(X, Y ; \psi_{1}, \psi_{2}\right) \geq 0$ and $G\left(X, Y ; \psi_{1}, \psi_{2}\right) \geq 0$ can be displayed as $\left(\langle i|X| i\rangle \equiv\left\langle\psi_{i}|X| \psi_{i}\right\rangle\right)$

$$
\begin{gathered}
\left(\Delta X\left(\psi_{1}\right)\right)^{2}\left(\Delta Y\left(\psi_{2}\right)\right)^{2} \geq\left|\left\langle\psi_{1}|(X-\langle 1|X| 1\rangle)(Y-\langle 2|Y| 2\rangle)| \psi_{2}\right\rangle\right|^{2}, \\
\left.\left.\left[\left(\Delta X\left(\psi_{1}\right)\right)^{2}+\langle 1|X| 1\rangle^{2}\right)\right]\left[\left(\Delta Y\left(\psi_{2}\right)\right)^{2}+\langle 2|Y| 2\rangle^{2}\right)\right] \geq\left|\left\langle\psi_{1}|X Y| \psi_{2}\right\rangle\right|^{2} .
\end{gathered}
$$


It is not difficult to establish (after some manipulations) that the inequality (19) is less precise than (18). The equalities in (18) and (19) are not invariant under linear transformations of observables and/or states. The equations (8) and (9) with $H_{1}=R\left(X, Y ; \psi_{1}\right)$ and $H_{2}=R\left(X, Y ; \psi_{2}\right)$ both produce an entangled but very compact $(2,2) \mathrm{UR}$,

$$
\begin{aligned}
\frac{1}{2}\left[\left(\Delta X\left(\psi_{1}\right)\right)^{2}\left(\Delta Y\left(\psi_{2}\right)\right)^{2}+\left(\Delta X\left(\psi_{2}\right)\right)^{2}\right. & \left.\left(\Delta Y\left(\psi_{1}\right)\right)^{2}\right]-\Delta X Y\left(\psi_{1}\right) \Delta X Y\left(\psi_{2}\right) \\
& \geq \frac{1}{4}\left\langle\psi_{1}|[X, Y]| \psi_{1}\right\rangle\left\langle\psi_{2}|[X, Y]| \psi_{2}\right\rangle^{*} .
\end{aligned}
$$

The equality in this relation is invariant under linear transformations of $X, Y$, but not of $\left|\psi_{1}\right\rangle$ and $\left|\psi_{2}\right\rangle$. With $\left|\psi_{1}\right\rangle=\left|\psi_{2}\right\rangle$ in 20 one recovers the Schrödinger UR (11). The inequality (20) should be referred to as state extended Schrödinger UR. For the canonical $p$ and $q$ it simplifies to

$$
\frac{1}{2}\left[\left(\Delta p\left(\psi_{1}\right)\right)^{2}\left(\Delta q\left(\psi_{2}\right)\right)^{2}+\left(\Delta p\left(\psi_{2}\right)\right)^{2}\left(\Delta q\left(\psi_{1}\right)\right)^{2}\right]-\Delta p q\left(\psi_{1}\right) \Delta p q\left(\psi_{2}\right) \geq \frac{1}{4} .
$$

Similar to (but less precise than) (20) is the $(2,2)$ UR obtained again from (8) and (9) with the third choice (14). The entangled UR (20) admits less precise version of the form (corresponding to $\triangle X Y=0)$

$$
\frac{1}{2}\left[\left(\Delta X\left(\psi_{1}\right)\right)^{2}\left(\Delta Y\left(\psi_{2}\right)\right)^{2}+\left(\Delta X\left(\psi_{2}\right)\right)^{2}\left(\Delta Y\left(\psi_{1}\right)\right)^{2}\right] \geq \frac{1}{4}\left|\left\langle\psi_{1}|[X, Y]| \psi_{1}\right\rangle\left\langle\psi_{2}|[X, Y]| \psi_{2}\right\rangle\right| .
$$

The latter inequality can be regarded as an entangled extensions of the Heisenberg UR. For $X=p$ and $Y=q$ the right hand side of (22) simplifies to $1 / 4$.

In view of the Remark 1 if UR (20) is minimized then (11) is saturated by $\left|\psi_{1}\right\rangle$ and $\left|\psi_{2}\right\rangle$. Therefore (20) can be used for finer classification of Schrödinger intelligent states. From any extended UR one can obtain new ordinary UR by fixing all but one of the states. For example if $\left|\psi_{1}\right\rangle$ in (21) is fixed as a canonical coherent state then (21) produces $(\Delta p)^{2}+(\Delta q)^{2} \geq 1$. The latter UR is minimized in canonical coherent states only, while the Heisenberg UR $(\Delta p)^{2}(\Delta q)^{2} \geq 1 / 4$ is minimized in any squeezed state with $\Delta p q=0$.

UR of type $(\mathbf{3}, \mathbf{1})$ and type $(\mathbf{2}, \mathbf{m}), m>2$. From higher order UR we note two cases: the case of $(3,1)$ - UR corresponding to the choice $H_{1}=G(X, Y ; \psi), H_{2}(X, Z ; \psi)$ in (9), and the case of $(2, \mathrm{~m})$ - UR corresponding to the choice $H_{\mu}=R\left(X, Y ; \rho_{\mu}\right)$ in the matrix inequality (9). After some consideration we arrive at the explicite formulas for two entangled UR,

$$
\begin{gathered}
(\Delta X)^{2}(\psi)\left[(\Delta Y(\psi))^{2}+(\Delta Z(\psi))^{2}\right] \geq 2 \Delta X Y(\psi) \Delta X Z(\psi)+\frac{1}{2}\langle\psi|[X, Z]| \psi\rangle\langle\psi|[Y, X]| \psi\rangle, \\
\sum_{\mu<\nu}^{m}\left[\left(\Delta X\left(\rho_{\mu}\right)\right)^{2}\left(\Delta Y\left(\rho_{\nu}\right)\right)^{2}+\left(\Delta X\left(\rho_{\nu}\right)\right)^{2}\left(\Delta X\left(\rho_{\mu}\right)\right)^{2}\right]-2 \sum_{\mu<\nu}^{m} \Delta X Y\left(\rho_{\mu}\right) \Delta Y Y\left(\rho_{\nu}\right) \\
\geq 2 \sum_{\mu<\nu}^{m} C\left(X, Y ; \rho_{\mu}\right) C\left(X, Y ; \rho_{\nu}\right),
\end{gathered}
$$

where $C(X, Y ; \rho)=-(i / 2)\langle[X, Y]\rangle_{\rho}=(-i / 2) \operatorname{Tr}(\rho[X, Y])$. With $Z=Y$ in (23) one gets Schrödinger UR (1) for $X, Y$, while $X=Y$ produces new ordinary UR $(\Delta X(\psi))^{2}+(\Delta Z(\psi))^{2} \geq 2 \Delta X Z(\psi)$. At $m=2$ the inequality (24) recovers (20).

\section{Conclusion}

We have established several classes of extended characteristic uncertainty relations (UR) of type $(\mathrm{n}, \mathrm{m})$ for $n$ observables and $m$ states using the Gram matrices of suitably constructed nonnormalized states. Entangled UR can be obtained using characteristic inequalities (9) and (8). 
The extended UR reveal global statistical correlations of quantum observables in distinct states. The characteristic inequalities could be useful in many fields of mathematical and quantum physics, in particular in the precise measurement theory. Extended UR can be used for construction of observable induced distances between quantum states and for finer classification of states, in particular of group-related coherent states.

\section{References}

[1] Heisenberg W. 1927, Z. für Phys. 43 172-198, Uber den anschaulichen Inhalt der quantentheoretishen Kinematik and Mechanik, Kennard E.H. 1927, Z. für Phys. 44 326-352, Zur Quantenmechanik einfacher Bewegungstypen.

[2] Schrödinger E. 1930, Sitz. Preus. Acad. Wiss. (Phys.-Math. Klasse), p 296-303, Zum Heisenbergschen Unschärfeprinzip,

Robertson H.P. 1930, Phys. Rev. 35 667-667, A general formulation of the uncertainty principle and its classical interpretation.

[3] Robertson H.P. 1934, Phys. Rev. 46 794-801, An indeterminacy relation for several observables and its classical interpretation.

[4] Loudon R. and Knight P. 1987, J. Mod. Opt. 34 709, Squeezed light.

[5] Dodonov V.V., Kurmyshev E.V. and Man'ko V.I. 1980, Phys. Lett. A 79 150, Generalized uncertainty relation an correlated coherent states.

[6] Wodkiewicz K. and Eberly J. 1985, J. Opt. Soc. Am. B 2 458, Coherent states, squeezed fluctuations and the $S U(2)$ and $S U(1,1)$ groups in quantum optics applications.

[7] Trifonov D.A. 1994, J. Math. Phys. 35 2297, Generalized intelligent states and squeezing, Trifonov D.A. 1993, Generalized intelligent states and $S U(1,1)$ and $S U(2)$ squeezing, Preprint INRNE-TH-93/4 (May 1993) quant-ph/0001028.

[8] Trifonov D.A. 1997, J. Phys. A: Math. Gen 30 5941, Robertson intelligent states, Trifonov D.A. 1998, Phys. Scripta 58 246, On the squeezed states for $n$ observables.

[9] Trifonov D.A. and Donev S.G. 1998, J. Phys. A: Math. Gen. 31 8041, Characteristic uncertainty relations.

[10] Simon R. and Mukunda N. 1997, Moments of the Wigner Distribution and a Generalized Uncertainty Principle, E-print quant-ph/9708037,

Santhanam T S 2000, J. Phys. A: Math. Gen. 33 L83, Higher-order uncertainty relations.

[11] Trifonov D.A. 1999, The uncertainty way of generalization of coherent states, E-print quant$\mathrm{ph} / 9912084$.

[12] Gantmaher F.R. 1975. Teoria matrits (Nauka, Moscow). 